\title{
Neonate with permanent neonatal diabetes mellitus; a very rare homozygous missense mutation in the glucokinase gene
}

\begin{abstract}
Permanent neonatal diabetes mellitus (PNDM) appears within the first six months of life. It represents $50 \%$ of cases of neonatal diabetes. Affected neonates present with hyperglycemia, intrauterine growth retardation, polyuria, dehydration, and failure to thrive.

PNDM requires lifelong insulin treatment leading to catch-up growth. The course varies according to the underlying causative gene. GCK mutations cause $4 \%$ of PNDM that present from the first day of life. Homozygous mutations in the glucokinase gene (GCK) causes PNDM while heterozygous GCK mutations cause maturity-onset diabetes of the young (MODY).

We report a male neonate of Arab ancestry who was delivered by cesarean section at 36 weeks gestation. He had intrauterine growth retardation with birth weight $1.720 \mathrm{~kg}$.

He presented in the first few days of life with hyperglycemia \{Serum glucose $>250$ $\mathrm{mg} / \mathrm{dl}(13.8 \mathrm{mmol} / \mathrm{l})\}$. On 10th day of life insulin treatment was started. The initial insulin requirement ranged from $0.05-0.3$ units per $\mathrm{Kg}$ body weight per day. Blood glucose control was difficult initially to be achieved with difficulties in preparing such small doses of insulin, and large variations in blood glucose concentrations but no ketosis.

His investigations revealed low serum insulin and C-peptide. The glycated hemoglobin (HBA1c) after birth was $4.8 \%$ which raised up to $6.1 \%$ at age of 2 months. Whole exome sequence revealed a very rare novel homozygous missense pathogenic variant c. $667 \mathrm{G}>\mathrm{A}$ (p.Gly223Ser) in the glucokinase gene. His parents are first cousins. His father has impaired fasting hyperglycemia that was treated with diet control. His mother has systemic lupus, primary hypothyroidism, protein $\mathrm{S}$ and anti-thrombin deficiency. She had gestational diabetes in all her pregnancies. Very strong family history of type 2 diabetes mellitus and MODY in his first-degree relatives.

To date, this missense p.Gly223Ser mutation has only been identified in heterozygous state in patients with GCK-MODY and type 2 diabetes, this is the first study to identify the homozygous form of this novel mutation in PNDM.
\end{abstract}

Abbreviations: MCT, medium chain triglyceride; IUGR, intrauterine growth restriction; USS, Ultrasound; SLE, systemic lupus erythematosus; SC, subcutaneous; NDM, neonatal diabetes mellitus; GCK, glucokinase

\section{Introduction}

While the early onset of NDM in the first six months of life rarely occurs as a result of autoimmune disorder, genetic mutations and chromosomal abnormalities are recognizable causes of NDM. ${ }^{1-}$ ${ }^{7}$ Mutations in KCNJ11 or ABCC8 gene, that encode for subunits of the ATP sensitive potassium channel, are the most commonly encountered mutations in permanent $\mathrm{NDM}^{8}$ Previous reports showed thet permanent NDM may result from mutations in insulin or glucokinase (GCK) genes as well. ${ }^{910}$ Homozygous GCK-related permanent NDM is associated with complete GCK deficiency and severe hyperglycemia. ${ }^{11,12}$

In this report, we presented a case of permanent NDM due to a very rare, novel homozygous missense mutation in GCK gene.

\section{Case report}

A male baby born to a second-degree consanguineous parent at
Volume 9 Issue I - 2019

\author{
Manal Mustafa,' Khaled El-Atawi, ${ }^{2}$ Ahmed \\ Zakaria, ${ }^{2}$ Mahmoud Elhalik, ${ }^{2}$ Fatma Bastaki ${ }^{3}$ \\ 'Pediatric Endocrinology Division, Department of Pediatrics, \\ Latifa Women and Children Hospital, UAE \\ ${ }^{2}$ Neonatal Intensive Care Unit, Latifa Women and Children \\ Hospital, UAE \\ ${ }^{3}$ Genetic \& Metabolic Division, Department of Pediatrics, Latifa \\ Women and Children Hospital, UAE
}

Correspondence: Khaled El-Atawi, Neonatal Intensive Care Unit, Latifa Women and Children Hospital, Dubai, UAE, Email kelatawi@eim.ae

Received: October II, 2018 | Published: February 04, 2019
36 weeks gestation with a low birth weight of $1.720 \mathrm{~kg}$ and APGAR score of 9 and 10 at 1 and 5 min respectively. Antenatal ultrasound (USS), showed intrauterine growth restriction (IUGR). Mother was 30 years old, $\mathrm{P} 3+4$, with multiple autoimmune disorders including hypothyroidism due to autoimmune thyroiditis, systemic lupus erythematosus (SLE), protein S deficiency, and anti-thrombin deficiency. In her previous pregnancies, she was diagnosed with gestational diabetes then she developed a permanent type 2 diabetes mellitus two months before this pregnancy. His father has mild fasting hyperglycemia controlled by diet. Strong family history of type II diabetes mellitus in first degree relatives (one maternal aunt, five maternal uncles, both maternal grandparents, paternal grandfather and four of father's paternal aunts). His male maternal cousin, who is currently 25 years old, had early onset diabetes during first month of life, controlled by insulin, but no confirmatory genetic test was done.

The baby's initial examination was normal apart from low birth weight. He underwent a routine blood glucose monitoring for hypoglycemia for the first 24 hours which showed mild hyperglycemia. From day 3 of life, the blood glucose level started rising more and ranged from $180-250 \mathrm{mg} / \mathrm{dl}(10-13.8 \mathrm{mmol} / \mathrm{l})$. This persistent hyperglycemia raised our index of suspicion of the possibility of neonatal diabetes especially after exclusion of other 
causes of hyperglycemia such as (sepsis and stress) especially in view of the family history, On day 9 of life, baby's investigations revealed glucosuria without ketonuria, Serum insulin $2.8 \mathrm{uIU} / \mathrm{ml}$ (4.0-16.0), C-peptide $0.4 \mathrm{ng} / \mathrm{ml}(1.8-4.7)$ when serum glucose was $218 \mathrm{mg} / \mathrm{dl}$ (12mmol/l). The HbAlc was $4.8 \%$ on day 5 , which raised up $6.1 \%$ at age of three months. The diagnosis of NDM (Neonatal Diabetes Mellitus) was raised by day 10 of life and the insulin was started in the form of intermediate acting insulin (Isophan insulin) with initial dose of 0.1 units subcutaneous (SC) daily $(0.05 \mathrm{iu} / \mathrm{kg} /$ day $)$. Whole exome sequence test was sent for the baby and his parents to delineate the underlying genetic cause.

On day 15 of life, blood glucose readings were still high; therefore, the Isophan insulin $(0.1$ units SC) was given twice daily $(0.1 \mathrm{iu} / \mathrm{kg} / \mathrm{day})$. Later, on day 30 of life, baby was started on multiple daily injections (MDI) by adding short acting insulin (Aspart) 0.1 units with $4^{\text {th }}$ hourly feeds. Insulin doses were frequently increased and adjusted according to blood sugar readings and feeds. Baby was reviewed regularly by dietician to optimize calories intake and ensure normal growth. It was a challenge to achieve the optimum growth with minimizing the hyperglycemia. Baby was started on high caloric feedings with low glucose content, that lead us to use of EBM (expressed mother milk) plus HMF (Human milk fortifiers) or preterm formula. Later, to achieve the high caloric requirements while maintaining the low glucose content, Medium Chain Triglyceride (MCT) oil was introduced with term formula or the breast milk aiming to maintain normal growth.

On day 74 of life, in view of hypoglycemia at the time of Isophane insulin peak action, we changed basal insulin to insulin Glargine 1 unit daily, with Aspart insulin $4^{\text {th }}$ hourly with feeding according to blood glucose level. He was discharged from the hospital at age of 3 months in good general condition, body weight of $4 \mathrm{~kg}$ and during follow up in pediatric diabetes clinic, insulin Aspart was changed to Lispro.

Whole exome sequence revealed a very rare homozygous missense pathogenic mutation c.667G $>$ A (p.Gly223Ser) in the GCK (glucokinase) gene. Both parents carried the same mutation in heterozygous state. Currently the baby is twelve months old, on multiple daily injections of SC insulin of Glargine and Lispro. $\mathrm{He}$ is thriving well with good blood sugar control and occasional hypoglycemia. His last $\mathrm{HbAlc}$ at age of twelve months was $7.1 \%$. $\mathrm{He}$ is on continuous glucose monitoring by Dexcom G5 device and planned for insulin pump.

\section{Discussion}

In this report, we describe a male baby with permanent NDM due to a very rare homozygous missense pathogenic mutation c. $667 \mathrm{G}>\mathrm{A}$ (p.Gly223Ser) in the GCK gene because of heterozygous mutations in both parents. Glycemic control was challenging, patient was treated with multiple daily injections of SC insulin and eventually performed well.

The role of genetic mutations in the development of permanent NDM is well established. GCK is a member of the hexokinase family which produce glucose-6-phosphate. Alteration in GCK level or function is associated with abnormalities in glucose metabolism and insulin secretion ${ }^{13,14}$. The current published literature showed few permanent NDM cases, with homozygous or compound heterozygous mutations, with decreased GCK activity, however, no common mutations have been identified yet ${ }^{15-18}$. Bennett and colleagues ${ }^{11}$ reported four cases of permanent NDM due to novel homozygous nonsense GCK mutation in exon 3 (Q98X) or missense GCK mutation in exon 7 (G261R), which were previously found in heterozygous state in GCK-MODY and type 2 diabetes only.

Gly223 residue is located in the $\beta$-sheet of the large domain hydrophobic core, the missense mutation in the present case resulted in the substitution of glycine with serine which may lead to defect in the GCK structure and activity. ${ }^{19,20}$ Given the homozygous form of the mutation in the reported case, the baby presented with severe hyperglycemia in the first few days of life, which required prompt insulin therapy, and gradual titration of its dose. The baby is currently treated with multiple daily injections of SC insulin. Homozygous GCK mutations were reported to be associated with severe hyperglycemia, and sometimes ketoacidosis, due to complete GCK deficiency; ${ }^{10,21}$ while heterozygous mutations were linked to mild form that rarely require treatment. ${ }^{4}$ Interestingly, Borowiec and colleagues reported a permanent NDM case presented with ketoacidosis in the first weeks of life due to heterozygous p.Gly223ser mutation in GCK. The authors attributed this severe form to the coexistence of potential additional genetic mutation or an interaction between different genetic factors. ${ }^{22}$

As mentioned, heterozygous mutations in GCK gene was linked to the development of MODY2, Capuano and colleagues reported 19 heterozygous GCK mutations in 28 children with suspected MODY $2 .^{23}$ In the present report, both parents carried the same mutation in heterozygous state, and the mother has established type 2 diabetes on insulin and metformin.

We faced many obstacles in order to achieve the glycemic control in our case. Being IUGR neonate with lack of subcutaneous fat, unpredictable insulin subcutaneous absorption with the rapid development of hypertrophy at insulin injection sites, made it difficult to administer the SC insulin. These challenges directed us to the use of I-port device with frequent change of the insulin injection sites in rotational manner until the growth had been achieved and subcutaneous fat had been well developed. To date, our reported missense p.Gly223Ser mutation has only been identified in heterozygous state in patients with GCK-MODY and type 2 diabetes; this is the first study to identify the homozygous form of this rare mutation in permanent NDM.

\section{Acknowledgments}

None.

\section{Conflict of interest}

The author declares no conflict of interest.

\section{References}

1. Greeley SAW, Tucker SE, Worrell HI, et al. Update in neonatal diabetes. Curr Opin Endocrinol Diabetes Obes. 2010;17:13-19.

2. Nansseu JRN, Ngo-Um SS, Balti EV. Incidence, prevalence and genetic determinants of neonatal diabetes mellitus: A systematic review and meta-analysis protocol. Syst Rev. 2016;5(1):188.

3. Rearson MA, Mc Knight-Menci H, Steinkrauss L. Neonatal diabetes: current trends in diagnosis and management. MCN Am J Matern Child Nurs. 2011;36:14-17.

4. Naylor RN, Greeley SAW, Bell GI, et al. Genetics and pathophysiology of neonatal diabetes mellitus. J Diabetes Investig. 2011;2:158-169. 
5. Aguilar-Bryan L, Bryan J. Neonatal diabetes mellitus. Endocr Rev. 2008;29:265-291.

6. Edghill EL, Dix RJ, Flanagan SE, et al. HLA genotyping supports a nonautoimmune etiology in patients diagnosed with diabetes under the age of 6 months. Diabetes. 2006;55:1895-1898.

7. Gach A, Wyka K, Pietrzak I, et al. Neonatal diabetes in a child positive for islet cell antibodies at onset and Kir6.2 activating mutation. Diabetes Res Clin Pract. 2009;86(2):e25-e27.

8. Ashcroft FM, Puljung MC, Vedovato N. Neonatal Diabetes and the KATPChannel: From Mutation to Therapy. Trends Endocrinol Metab. 2017;28:377-387.

9. Støy J, Edghill EL, Flanagan SE, et al. Insulin gene mutations as a cause of permanent neonatal diabetes. Proc Natl Acad Sci U S A. 2007;104:15040-15044.

10. Njølstad PR, Sagen JV, Bjørkhaug L, et al. Permanent Neonatal Diabetes Caused by Glucokinase Deficiency: Inborn Error of the Glucose-Insulin Signaling Pathway. Diabetes. 2003;52:2854-2860.

11. Bennett K, James C, Mutair A, et al. Four novel cases of permanent neonatal diabetes mellitus caused by homozygous mutations in the glucokinase gene. Pediatr Diabetes. 2011;12:192-196.

12. Hussain K. Mutations in pancreatic -cell Glucokinase as a cause of hyperinsulinaemic hypoglycaemia and neonatal diabetes mellitus. Rev Endocr Metab Disord. 2010;11:179-183.

13. Gidh-Jain M, Takeda J, Xu LZ, et al. Glucokinase mutations associated with non-insulin-dependent (type 2) diabetes mellitus have decreased enzymatic activity: implications for structure/function relationships. Proc Natl Acad Sci U S A. 1993;90:1932-1936.

14. Davis EA, Cuesta-Muñoz A, Raoul M, et al. Mutants of glucokinase cause hypoglycaemia- and hyperglycaemia syndromes and their analysis illuminates fundamental quantitative concepts of glucose homeostasis. Diabetologia. 1999;42:1175-1186.
15. Porter JR, Shaw NJ, Barrett TG, et al. Permanent neonatal diabetes in an Asian infant. J Pediatr. 2005;146:131-133.

16. Njølstad PR, Søvik O, Cuesta-Muñoz A, et al. Neonatal Diabetes Mellitus Due to Complete Glucokinase Deficiency. $N$ Engl $J$ Med. 2001;344:1588-1592.

17. Turkkahraman D, Bircan I, Tribble ND, et al. Permanent Neonatal Diabetes Mellitus Caused by a Novel Homozygous (T168A) Glucokinase (GCK) Mutation: Initial Response to Oral Sulphonylurea Therapy. J Pediatr. 2008;153:122-126.

18. Rubio-Cabezas O, González FD, Aragonés A, et al. Permanent neonatal diabetes caused by a homozygous nonsense mutation in the glucokinase gene. Pediatr Diabetes. 2008;9:245-249.

19. García-Herrero CM, Galán M, Vincent O, et al. Functional analysis of human glucokinase gene mutations causing MODY2: exploring the regulatory mechanisms of glucokinase activity. Diabetologia. 2007; 50:325-333.

20. Massa O, Meschi F, Cuesta-Munoz A, et al. High prevalence of glucokinase mutations in Italian children with MODY. Influence on glucose tolerance, first-phase insulin response, insulin sensitivity and BMI. Diabetologia. 2001;44:898-905.

21. Velho G, Froguel P. Genetic, metabolic and clinical characteristics of maturity onset diabetics of the young. Eur J Endocrinol. 1998;138:233239.

22. Borowiec M, Mysliwiec M, Fendler W, et al. Phenotype variability and neonatal diabetes in a large family with heterozygous mutation of the glucokinase gene. Acta Diabetol. 2011;48:203-208.

23. Capuano M, Garcia-Herrero CM, Tinto N, et al. Glucokinase (GCK) mutations and their characterization in MODY2 children of Southern italy. PLoS One. 2012;7(6):e38906. 